\title{
Répartition spatiale de la végétation herbacée autour des mares temporaires : influence des facteurs anthropiques et topographiques à WIDOU THIENGOLY (Ferlo, Nord-Sénégal)
}

\author{
Ndiabou FAYE ${ }^{1}$, Aly DIALLO ${ }^{2}$, Moustapha Bassimbé SAGNA ${ }^{1}$, Omar SARR ${ }^{1}$, \\ Ramata TALLA ${ }^{1}$, Eric Sylvain BADJI ${ }^{1}$, Jean Luc PEIRY ${ }^{3}$ et Aliou GUISSE ${ }^{5 *}$ \\ ${ }^{1}$ Université Cheikh Anta Diop de Dakar Département de Biologie végétale, B.P. 5005, Dakar, Sénégal. \\ ${ }^{2}$ Université Assane Seck de Ziguinchor, Département d'agroforesterie, PO Box: 523 Néma, Ziguinchor, \\ Sénégal. \\ ${ }^{3}$ Université Clermont Auvergne (France), 63001 Clermont-Ferrand, cedex 1, France. \\ ${ }^{4}$ Observatoire Homme Milieu Tessékéré, CNRS/Université Cheikh Anta Diop, Dakar, Sénégal. \\ ${ }^{5}$ Unité Mixte Internationale 3189 « Environnement, Santé, Société » (UCAD, CNRS, CNRST, USTT, UGB), \\ Dakar, Sénégal. \\ *Auteur correspondant, E-mail: aliou.guisse@ucad.edu.sn
}

\section{REMERCIEMENTS}

Cette recherche a été financée par l'UMI3189 " Environnement, Santé, Sociétés " (UCAD, CNRS, CNRST, USTTB, UGB) et le Labex DRIIHM, programme " Investissements d'avenir " avec la référence ANR11-LABX-0010.

\section{RESUME}

Les mares présentent un grand intérêt environnemental en raison des services écosystémiques qu'elles fournissent. Au Ferlo, les activités pastorales sont développées autour des mares. La connaissance des ressources herbacées est importante pour une gestion durable du pâturage autour des mares temporaires. L'objectif de cette étude est de déterminer la répartition spatiale des ressources herbacées autour des mares par une approche phytoécologique. L'échantillonnage sur des transects est réalisé dans les différents faciès morpho-pédologiques de deux mares pâturées et une mare non pâturée. La flore herbacée recensée compte 55 espèces, réparties dans 41 genres dont 22 familles. Les familles des Poacées et des Fabacées sont plus fréquentes quelle que soit l'unité morpho-pédologique et la mare, suivi de la famille des Cypéracées dans les mares et des versants et la famille des Zygophyllacées dont l'espèce Tribulus terrestris sur les dunes. Le recouvrement est globalement élevé $(82,6 \%)$ dans la mare non pâturée et moyen sur les mares pâturées $(55,33 \%$ et $46,4 \%)$. Au niveau des versants, les espèces sont de grandes tailles, rares et très diversifiées. Les espèces vivaces sont plus fréquentes autour des mares pâturées alors que les espèces rares se retrouvent autour de la mare non pâturée. Ces résultats révèlent ainsi l'influence prédominante des facteurs anthropiques et topographiques sur la répartition spatiale de la végétation herbacée.

(C) 2020 International Formulae Group. All rights reserved.

Mots clés : Transects, pâturée, phytoécologique, morpho-pédologiques. 


\title{
Distribution of herbaceous vegetation around temporary ponds: influence of anthropic and topographical factors in Widou Thiengoly (Ferlo, Northern Senegal)
}

\begin{abstract}
At the Ferlo, pastoral activities are developed around the ponds. The knowledge of herbaceous resources is important for sustainable grazing management around the temporary pools. The general objective of this study is to determine the spatial distribution of herbaceous resources around the ponds using a phytoecological approach. Transect sampling was carried out in the different morpho-pedological facies of two grazed ponds and one ungrazed pond. The recorded herbaceous flora includes 55 species, divided into 41 genera including 22 families. The Poaceae and Fabaceae families are more frequent whatever the morpho-pedological unit and the pond, followed by the Cyperaceae family in the ponds and on the slopes and the Zygophyllaceae family including the species Tribulus terrestris on the dunes. The overall coverage is high $(82.6 \%)$ in the ungrazed pond and medium on the grazed ponds $(55.33 \%$ and $46.4 \%)$. At the level of the slopes, the species are large, rare and much diversified. Perennial species are more frequent around the grazed ponds, while rare species are found around the ungrazed pond. These results thus reveal the predominant influence of anthropogenic and topographical factors on the spatial distribution of herbaceous vegetation.
\end{abstract}

(C) 2020 International Formulae Group. All rights reserved.

Keywords: Transect, grazed, phytoecological, morpho-pedological.

\section{INTRODUCTION}

La ressource fourragère pour le cheptel est constituée par les végétations herbacée et ligneuse accessibles aux bétails. Au sahel, les pâturages naturels jouent un rôle important dans l'alimentation du bétail et constituent la base et, le plus souvent, la totalité des ressources alimentaires des animaux en élevage (Ousseina et al., 2013).

$\mathrm{Au}$ Ferlo, dans la région semi-aride du sahel, l'activité principale est le pastoralisme. Les fourrages herbacés et ligneux deviennent importants, voire indispensables pour la survie du cheptel pendant toute l'année (Akpo \& Grouzis, 2004 ; Ndiaye et al., 2010 ; Diallo et al., 2011; Ngom et al., 2012 ; Ousseina et al., 2013; Sarr et al., 2013 ; Niang et al., 2014 ; Ndiaye, 2015; Ndiaye et al., 2017). Dans cette région, le facteur le plus limitant pour la croissance des plantes est la teneur en eau de la zone d'enracinement (Pierre et al., 2011 ; Cissé et al., 2015).

La forte variabilité des précipitations dans le temps et dans l'espace (Ndiaye, 2015) conditionne la productivité des pâturages surtout au niveau des mares temporaires où les activités pastorales sont particulièrement développées (Diop et al., 2004). La courte saison des pluies, l'amenuisement des ressources herbacées dès la fin de ladite saison et l'utilité des mares dans la conservation de l'eau à plus ou moins moyen terme et dans la conservation de la strate herbacée conduisent à la recherche des connaissances sur la disposition des ressources végétales herbacées aussi bien sur le plan quantitatif que qualitatif. Donc cette étude est d'une importance capitale pour une amélioration de la productivité et une gestion durable du pâturage autour des mares temporaires.

Elle vise à évaluer la composition, la répartition spatiale et la structure de la végétation herbacée autour des mares temporaires sous l'influence des gradients anthropique et topographique (toposéquence) de deux zones, une pâturée et une autre non pâturée. Le travail repose sur les hypothèses selon lesquelles les mares offrent des conditions favorables à l'installation de la strate herbacée. Cette dernière est aussi sujette aux facteurs topographiques et anthropiques.

\section{MATERIEL ET METHODES}

\section{Présentation de la zone d'étude}

L'étude a été effectuée au centre du Ferlo, dans le Nord Sénégal à la station de 
WIDOU THIENGOLY $\left(15^{\circ} 58^{\prime} 30^{\prime \prime} \mathrm{N}\right.$ et $\left.15^{\circ} 17^{\prime} 90^{\prime \prime} \mathrm{O}\right)$ sur la période allant du mois d'août 2018 au mois de septembre 2019 (Figure 1). Le climat de WIDOU THIENGOLY est de type sahélien aride, caractérisé par l'alternance d'une longue saison sèche qui dure neuf mois (d'octobre à juin) et d'une courte saison pluvieuse de trois mois (de juillet à septembre) (Niang et al., 2014). Avec la situation actuelle marquée par une baisse de la pluviométrie (Figure 2), la station se situe entre les isohyètes 300 et $400 \mathrm{~mm}$. La pluviométrie varie d'une année à l'autre avec une moyenne de $371,67 \mathrm{~mm}$ au cours des cinquante dernières années (données pluviométriques de la station météo du "Centre de recherches zootechniques de Dahra") (Ndiaye et al. 2014). La température moyenne oscille autour de $27,73{ }^{\circ} \mathrm{C}$ (Ndiaye et al. 2013).

Le relief de la zone d'étude est peu accidenté avec des sols subarides tropicaux et brun-rouge formés de matériaux sableux pauvres en argile (Ndiaye et al., 2013). L'absence de reliefs accidentés limite le développement du réseau hydrographique qui ne présente aucune trace d'écoulement organisé. Il est caractérisé en saison des pluies par la présence d'une multitude de petites mares endoréiques.

La végétation se présente sous la forme d'une steppe arbustive à arborée. C'est une formation mixte constituée d'arbres et d'arbustes épais, généralement épineux et d'un tapis discontinu d'espèces annuelles où dominent les Graminées pendant la saison des pluies (Cissé et al. 2015). La strate arborée est marquée par la forte présence de Balanites aegyptiaca ((L.) Del.) qui constitue parfois des peuplements mono-spécifiques, par la présence d'Acacia tortilis raddiana (Forst.) et Acacia senegal (L.) alors que la strate arbustive est constituée de Calotropis procera (Ait.) et Boscia senegalensis (Pers.). Quant à la strate herbacée, les espèces telles que Alysicarpus ovalifolius (Schumach. \& Thonn.) J. Léonard, Aristida mutabilis Trin. \& Rupr, Cenchrus bifloris Roxb, Digitaria horizontalis Willd, Eragrostis tremula (Lam.) Hochst. ex Steud, Schoenefeldia gracilis Kunth, Tribulus terrestris auct. et Zornia glochidiata Rchb. ex
DC sont les plus fréquentes (Bakhoum, 2013 ; Diallo et al., 2011)

\section{Choix des mares}

Le choix des mares est basé sur deux critères essentiels : le types d'activité développé vu que les mares son utilisées différemment $(\mathrm{Sy}, 2009)$ et le type d'aménagement. Sur cette base trois mares ont été choisies : WENDOU MARODI ou mare aux lions, BELEL SOW et BELEL LOUGAL ou mare à Trou (puits superficiels artisanaux) (Figure 3).

WENDOU MARODI est une mare où le degré de l'anthropisation est très faible. Elle a été clôturée depuis des décennies pour abriter les activités du projet allemand (le Projet sénégalo-allemand de reboisement de la zone nord avait pour objectif de restaurer et valoriser l'environnement à travers des programmes de reboisement de grande envergure (KA, 2016). En 2018 la clôture a été renforcée par le projet de la Grande Muraille Verte.

BELEL SOW est une mare privée appartenant à un vieux du village Belel Sow. Elle est très protégée mais l'action anthropique est très régulière puisque le propriétaire a un grand cheptel.

BELEL LOUGAL a été creusé par la population locale pour exploiter l'eau d'une petite nappe alluviale superficielle. C'est une mare pâturée certes mais l'accessibilité aux animaux est très limitée par les trous.

\section{Méthode d'inventaire des herbacées}

La végétation herbacée a été étudiée suivant une approche phytoécologique (Fournet \& Monestiez, 1987 ; Diallo et al., 2009). Au niveau de chaque mare, l'échantillonnage a été réalisé le long de deux (2) transects séquents disposés des zones exondées vers les zones inondées. L'un est perpendiculaire à l'axe d'écoulement des eaux de ruissellement dans la mare et l'autre disposé longitudinalement de l'extrémité amont de la mare en son centre. Ce qui fait au total 6 transects dans les trois mares (Figure 3).

Les transects phytoécologiques permettent d'identifier et d'illustrer des portions de transects homogènes et de 
déterminer leur composition floristique, afin de mieux comprendre l'organisation de la végétation le long des transects. Cette méthode permet ainsi une visualisation plus simple des données des transects, résumant l'information de tous les points et toutes les espèces à une série de séquences pour lesquelles on connaît les espèces principales. Ainsi sur chaque transect, le relevé s'effectue sur des séquences homogènes ou des groupements que l'on identifie le long de la ligne sur le terrain. La longueur des transects et des séquences sont variables suivant le changement des facteurs biotiques et abiotiques.

Sur chaque séquence nous avons inventorié les espèces végétales herbacées présentes, déterminé leur recouvrement et leur hauteur moyenne. Certains facteurs abiotiques tels que la pente (par l'application angle mètre), le degré d'humidité, l'orientation des écoulements, la texture de sol (par le test du malaxage entre le pouce) entres autres ont été relevés. Le prélèvement de sol est aussi effectué sur chaque séquence en profondeur 0 $20 \mathrm{~cm}$ afin de déterminer l'acidité de sol au laboratoire.

\section{Analyse statistique}

Les informations recueillies sur le terrain ont servi de base pour la description de la structure spatiale de la végétation herbacée. Elles sont saisies et traitées à l'aide du tableur Excel. Plusieurs paramètres tels que la richesse spécifique, la fréquence spécifique, la contribution spécifique, le recouvrement et la structure spécifique ont été soumis à un test d'anova et décrits. La distribution spatiale des espèces est analysée à l'aide de l'indice de similitude (indice de Jaccard $\left(\mathrm{I}_{\mathrm{j}}\right)$ ) qui indique la façon dont les espèces sont réparties dans l'espace. L'influence des variables écologiques sur la répartition spatiale de la végétation herbacée est analysée à l'aide des transects phytoécologiques.

\section{$\mathrm{I}_{\mathrm{j}}=\frac{N c}{(N 1+N 2)-N c}$}

$\mathrm{Nc}$ est le nombre d'espèces communes aux différents zones, $\mathrm{S} 1$ et $\mathrm{S} 2$ sont respectivement le nombre d'espèces des zone 1 et 2 . L'indice varie de 0 quand il n'existe aucune espèce commune entre les deux habitats, à 1 quand toutes les espèces sont communes des deux milieux.

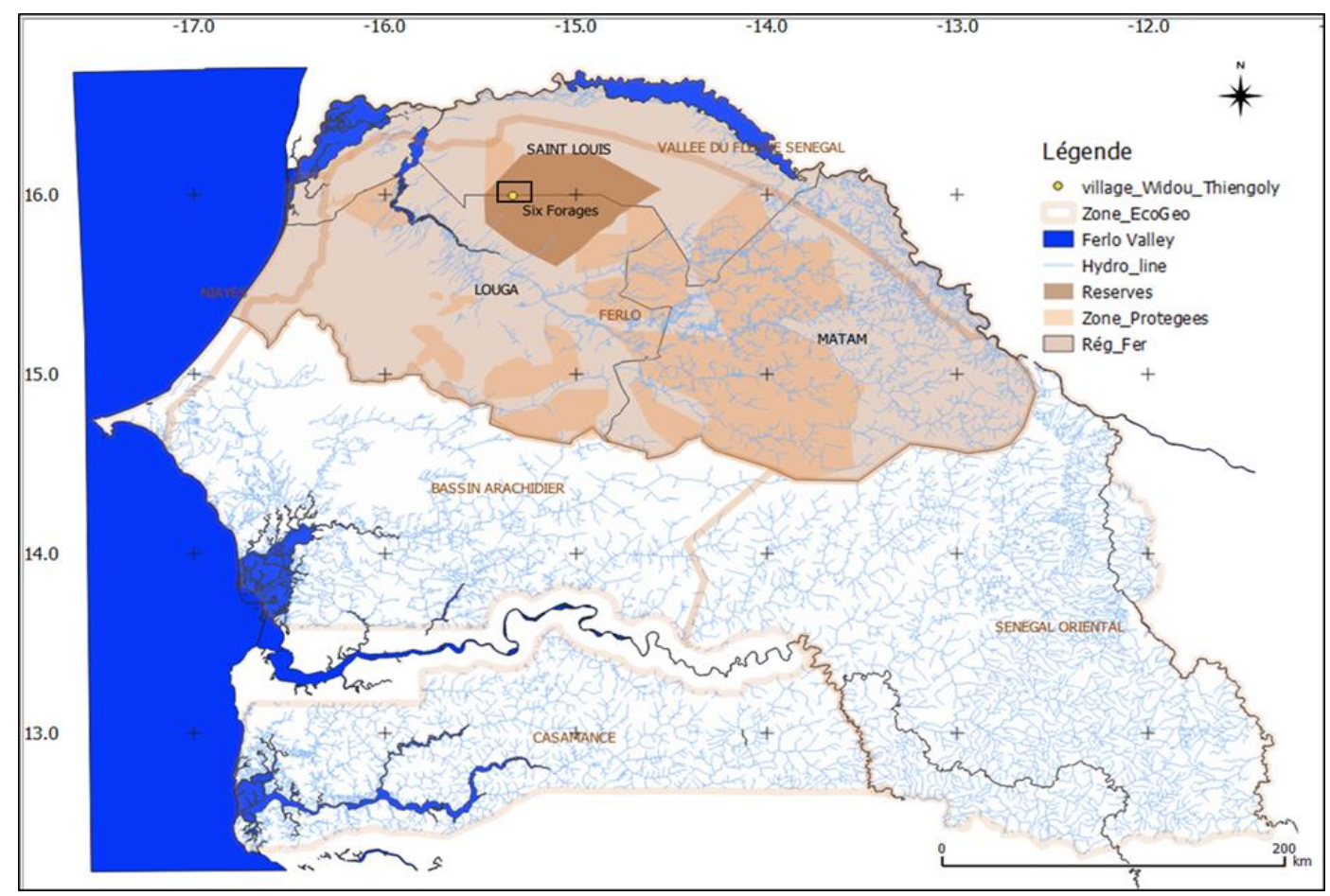

Figure 1 : Localisation de la zone d'étude. 


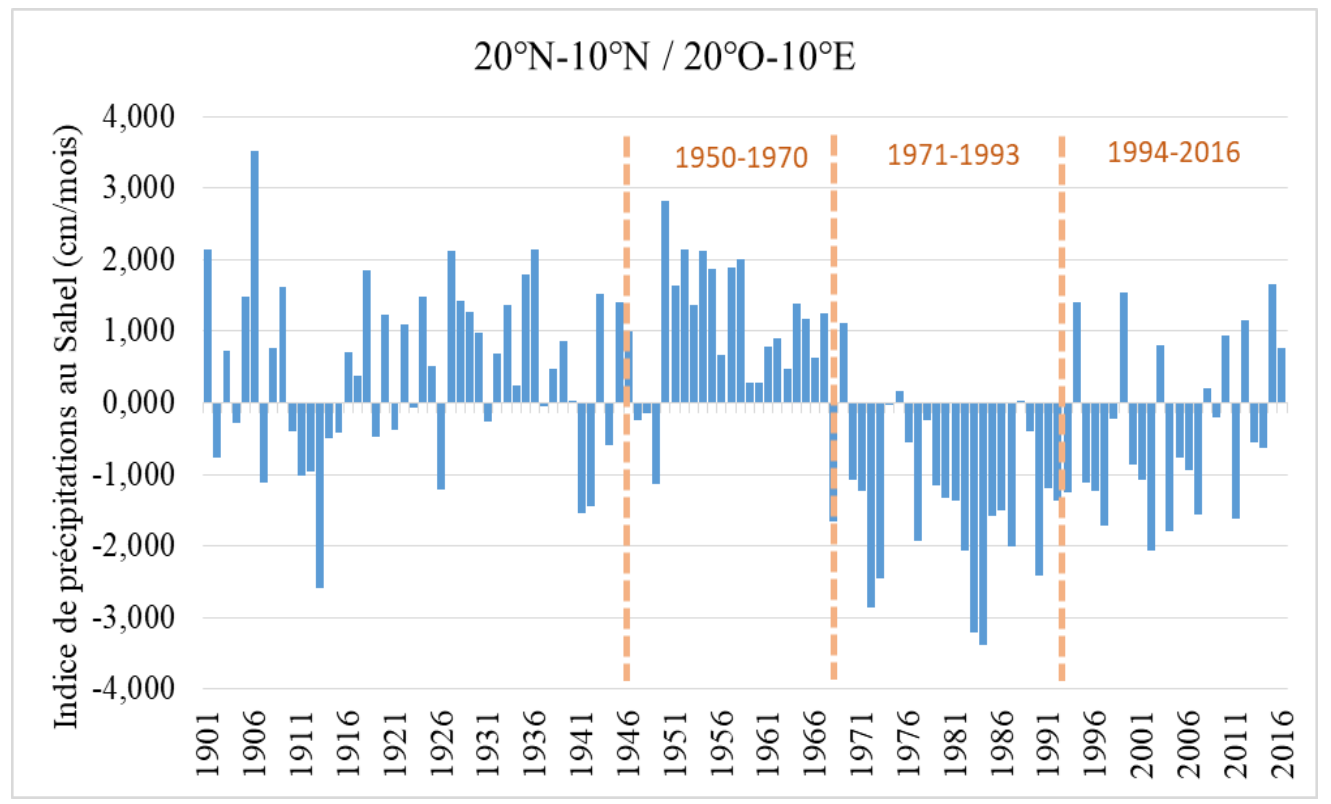

Figure 2 : Indice de précipitation au sahel

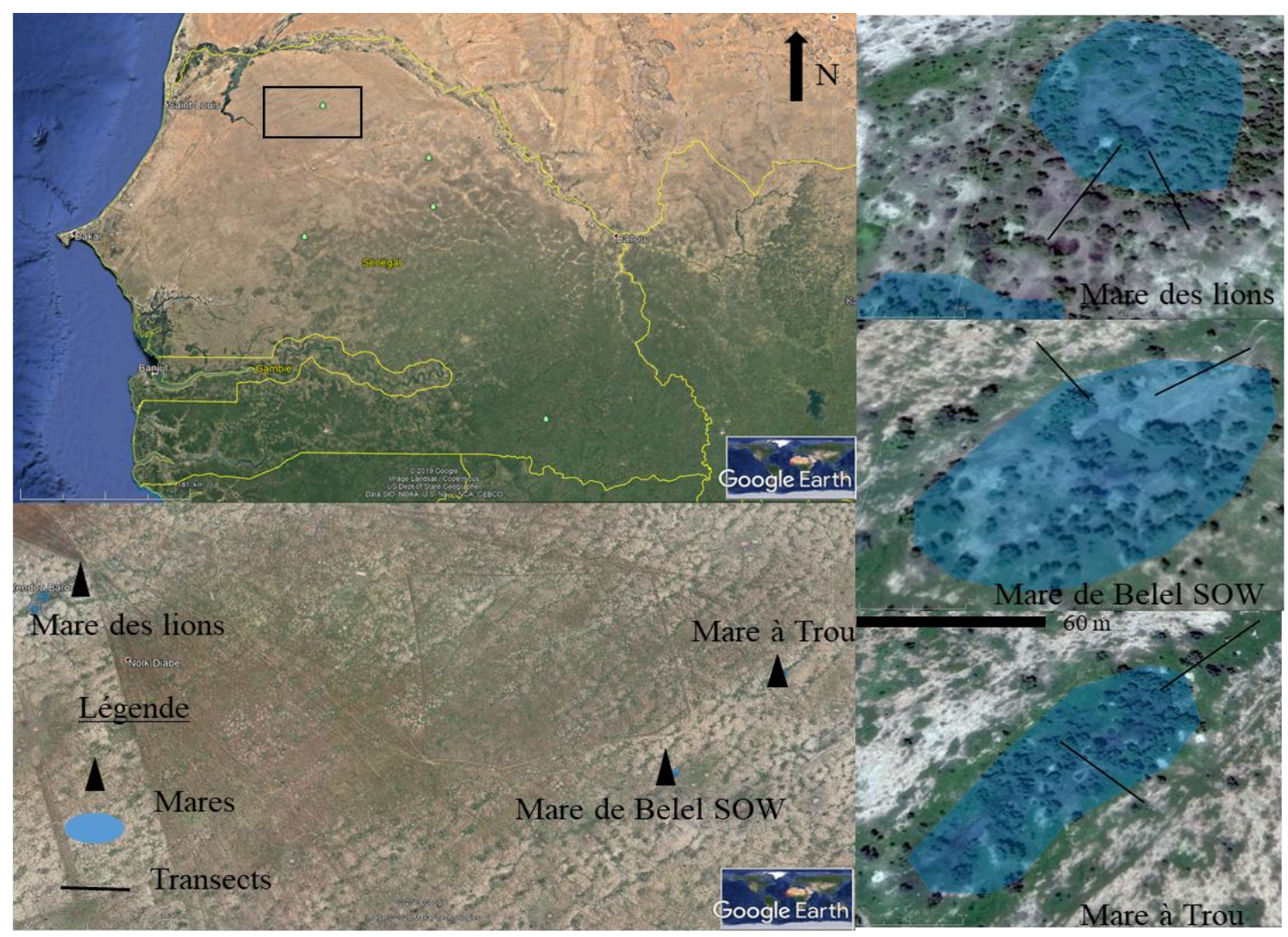

Figure 3 : Carte de localisation des mares et les transects (Faye, 2020). 


\section{RESULTATS}

\section{Composition et caractéristiques structurales de la végétation herbacée de la zone et des différentes mares}

La flore herbacée inventoriée dans les trois mares est riche de 55 espèces. Parmi ces espèces, les 31 réparties dans 27 genres et 17 familles ont été retrouvées dans et autour de la mare de Belel Sow ; les 42 réparties dans 33 genres et 15 familles de la mare à Trou et 49 dont 41 genres et 22 familles de la mare aux lions (Figure 4).

L'importance de chaque famille en termes de nombre de genres et d'espèces, de fréquence spécifique (Fs), de contribution spécifique (Cs) et de recouvrement spécifique (Rs) dans les zones d'étude et dans les trois mares a été présenté par le tableau 1. Les familles des Poacées et des Fabacées sont les mieux représentées dans la zone, quelle que soit la mare considérée en termes de genres et d'espèces. En tenant compte les contributions spécifiques et le recouvrement, ces deux familles dominent le peuplement herbacé suivi de la famille des Cypéracées et la famille des Rubiacées avec respectivement une contribution spécifique de $8,2 \%$ et $5,4 \%$. Les espèces provenant de la famille des Aracées, la famille des Commelinacées, la famille des Pedaliacées et la famille des Capparacées sont rares. Elles ont une contribution spécifique et un recouvrement inférieur respectivement à $0,5 \%$ et $0,05 \%$. Ces espèces ont été retrouvées uniquement dans la mare non pâturée (mare aux lions). D'ailleurs en considérant les différentes mares, la mare aux lions qui renferme le plus grand nombre de taxons (47 espèces et 36 genres) a en plus les 4 premières familles qui dominent le peuplement, la famille des Amaranthacées, la famille des Malvacées, la famille des Phyllanthacées et la famille des Acanthacées avec une bonne contribution spécifique. Quant aux mares à Trou et Belel Sow, la famille des Zygophyllacées et la famille des Nyctaginacées présentent une bonne contribution (Tableau 1). En termes de recouvrement et de contribution spécifique ces trois mares sont significativement différentes au seuil de 5\% (Tableau 2).

Le Tableau 3 présente plus d'information quant aux recouvrements et la contribution de chaque espèce dans les trois mares.

Les espèces Limeum indicum, Stylochaeton hypogaeus Lepr, Momordica charantia L., Corchorus aestuans, Commelina forsskaolii Vahl, Amaranthus hybridus L., Cleome viscosa L., Kilinga brevifolia, Sesamum $s p$ constituent les espèces rares dans la zone avec une contribution spécifique inférieur à $0,4 \%$. Ces espèces ont été retrouvées dans la mare aux lions hormis Kilinga brevifolia et Momordica charantia recensées dans la mare à Trou et Amaranthus hybridus $L$. dans la mare de Belel Sow. Peristrophe bicalyculata, Achyranthes aspera, Brachiaria Lata, Corchorus sp et Spermacoce sp ont été retrouvées seulement dans la mare aux lions tandis que les espèces Fimbristylis hispidula et Oldenlandia linearis sont spécifiques de la mare à Trou avec des contributions supérieures à $0,5 \%$.

Les autres espèces ont une contribution inférieure à $0,5 \%$ dans la zone et sont représentées au moins dans deux mares. Néanmoins les espèces qui dominent dans la zone restent Eragrostis tremula; Cyperus esculentus L., Indigofera hirsuta L.; Zornia glochidiata; Tribulus terrestris avec des contributions supérieures à 5\% (Tableau 3).

\section{Composition et caractéristiques structurales de la végétation herbacée des différentes unités topographiques}

En considérant les différentes unités topographiques, le recouvrement herbacé varie de $63,32 \%$ au niveau de la mare, $73,09 \%$ sur les versants et $46,68 \%$ sur les dunes. Quant au nombre de taxons, il est beaucoup plus important au niveau des versants (52 espèces et 32 genres) (Tableau 4). L'analyse comparative révèle une différence significative au seuil de $5 \%$ des différentes unités topographique en termes de contribution et de recouvrement (Tableau 5).

\section{Diversité floristique différentes mares et des différentes unités topographiques}

L'indice de similitude entre la mare aux lions et la mare à Trou est 0,69. Cela indique qu'un nombre important d'espèces se rencontre dans les deux zones donc la différence de biodiversité entre les habitats est faible. En 
revanche l'indice de similitude entre la mare aux lions et la mare de Belel Sow est faible $(0,5)$ évoquant ainsi que les mares ont peu d'espèces communes (Tableau 6).

Ces indices prouvent des conditions environnementales similaires entre la mare aux lions et la mare à Trou et des différences déterminantes des conditions environnementales entre la mare aux lions et la mare de Belel Sow.

A l'échelle des mares, les indices obtenus montrent que les espèces retrouvées dans les fonds mares et dans les inters dunes sont totalement différentes $(0,42$ pour la mare aux lions et 0,54 pour la mare à Trou) alors qu'un nombre important d'espèces sont communes des zones de transition et l'inter dune dans la mare de Sow et entre la zone de transition et le fond mare de la mare à Trou.

\section{Dynamique spatiale de la richesse floristique suivant les mares}

Une mise en parallèle des séquences et des pentes permet d'analyser le rôle de la géomorphologie dans la succession des espèces le long du transect (Fig. 5, 6,7).

Le transect de la mare à Trou présente trois zones qui se comportent différemment (Fond mare, zone de transition et les dunes). Sur la troisième zone, la diversité est importante mais leur recouvrement est faible
$(<10 \%)$ et que les espèces sont de très petites tailles. En revanche dans les zones de transition et le fond mare, les espèces ont un fort recouvrement et elles sont de grandes tailles. Les espèces qui colonisent ces zones sont Cyperus esculentus, Echinocholoa colona et Eragrostis tremula. C'est la mare qui reflète plus la situation qui sévit dans les mares de WIDOU THIENGOLY puisqu'elle est certes ouverte au publique mais très bien protégée par la population (Figure 5).

Les espèces retrouvées sur ce transect sont diversifiées, abondantes et de grandes tailles. Cette mare présente les conditions favorables à l'installation de plusieurs espèces herbacées. C'est une mare non pâturée où la végétation ligneuse est très développée surtout au niveau de la zone de transition. Une forte présence de l'Achyranthes $s p$ et Senna obtusifolia est observée dans la zone de transition où il $\mathrm{y}$ a un fort recouvrement ligneux (Figure 6).

Pour le transect de la mare de Belel Sow, le recouvrement végétal est très faible dans le fond mare $(\leq 10 \%)$ et moyen sur la zone et sur les dunes $(\leq 70 \%)$. Les espèces Tribulus terrestris, Zornia glochidiata, Alysicarpus ovalifolius, Dactyloctenium aegyptium, Indigofera hirsuta et Gisekia pharnaceoides colonisent la zone de transition et la dune (Figure 7).

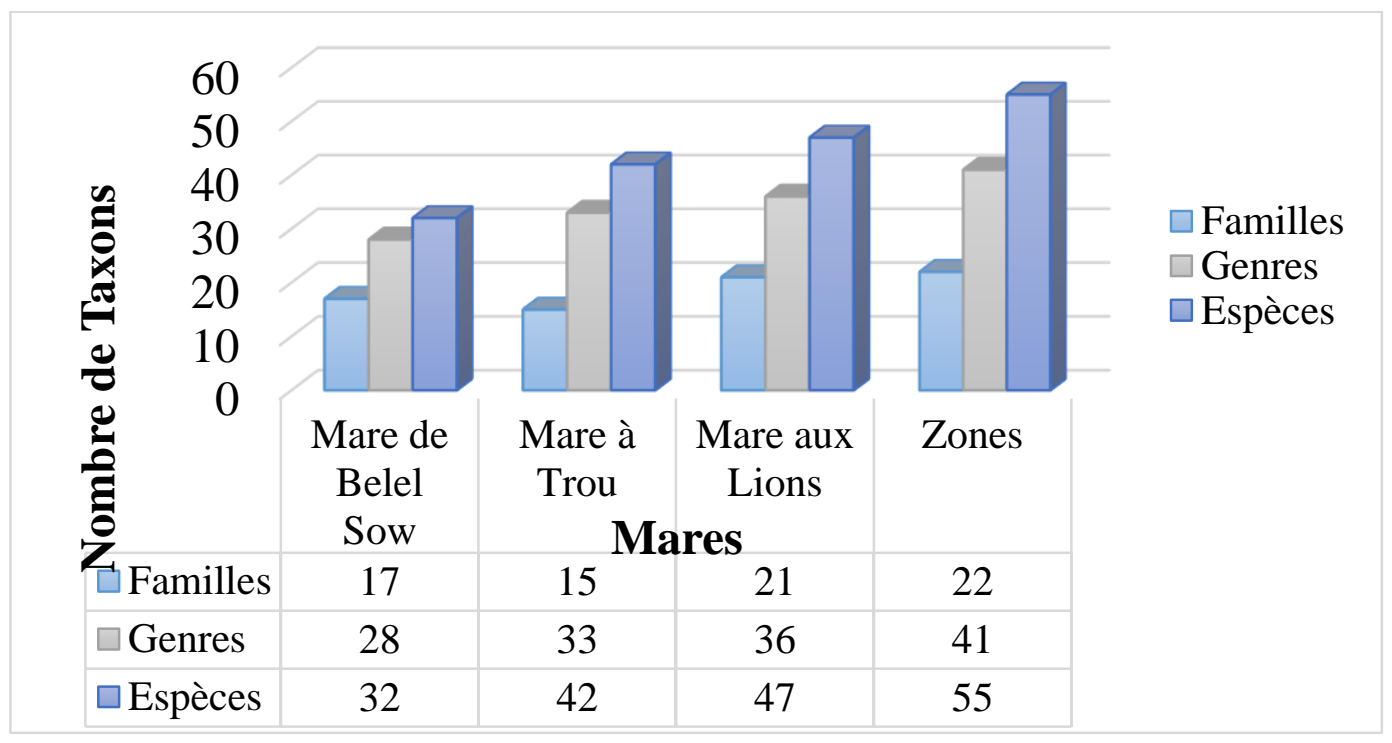

Figure 4 : La répartition taxonomique. 
Tableau 1 : Nombre de genres et espèces, fréquences, contributions et recouvrements spécifiques des différentes familles recensées

\begin{tabular}{|c|c|c|c|c|c|c|c|c|c|c|c|c|c|c|c|c|c|c|c|c|}
\hline \multirow{3}{*}{$\begin{array}{l}\text { Années } \\
\text { Familles }\end{array}$} & \multicolumn{5}{|c|}{ Zones } & \multicolumn{5}{|c|}{ Mare aux lions } & \multicolumn{5}{|c|}{ Mare à Trou } & \multicolumn{5}{|c|}{ Mare de Belel Sow } \\
\hline & \multicolumn{2}{|c|}{ Nombre de } & \multirow[t]{2}{*}{ Fs } & \multirow[t]{2}{*}{$\mathrm{Cs} \%$} & \multirow[t]{2}{*}{$\operatorname{Rs} \%$} & \multicolumn{2}{|c|}{ Nombre de } & \multirow[t]{2}{*}{ Fs } & \multirow{2}{*}{\multicolumn{2}{|c|}{ Cs \% Rs \% }} & \multicolumn{2}{|c|}{ Nombre de } & \multirow[t]{2}{*}{ Fs } & \multirow[t]{2}{*}{ Cs \% } & \multirow[t]{2}{*}{ Rs \% } & \multicolumn{2}{|c|}{ Nombre de } & \multirow[t]{2}{*}{ Fs } & \multirow[t]{2}{*}{ Cs \% } & \multirow[t]{2}{*}{ Rs \% } \\
\hline & $\mathbf{G}$ & $\mathbf{E}$ & & & & $\mathbf{G}$ & $\mathbf{E}$ & & & & G & $\mathbf{E}$ & & & & $\mathbf{G}$ & $\mathbf{E}$ & & & \\
\hline Poaceae & 12 & 15 & 494 & 37,7 & 34,8 & 12 & 15 & 194 & 34,1 & 45,4 & 12 & 14 & 226 & 42,5 & 33,07 & 9 & 10 & 90 & 34,4 & 20,5 \\
\hline Fabaceae & 4 & 4 & 256 & 19,5 & 8 & 4 & 4 & 107 & 18,8 & 7,42 & 4 & 4 & 113 & 21,2 & 11,55 & 3 & 3 & 42 & 16,0 & 6,19 \\
\hline Cyperaceae & 3 & 3 & 107 & 8,2 & 4,99 & 1 & 1 & 46 & 8,1 & 6,09 & 3 & 3 & 43 & 8,1 & 4,12 & 1 & 1 & 23 & 8,8 & 5,78 \\
\hline Rubiaceae & 2 & 5 & 71 & 5,4 & 2,34 & 2 & 4 & 49 & 8,6 & 5,31 & 2 & 5 & 21 & 3,9 & 0,35 & 1 & 2 & 20 & 7,6 & 0,09 \\
\hline Amaranthaceae & 2 & 3 & 28 & 2,1 & 2,65 & 1 & 2 & 27 & 4,7 & 6,4 & 0 & 0 & 0 & 0,0 & 0 & 1 & 1 & 1 & 0,0 & 0,03 \\
\hline Cucurbitaceae & 2 & 2 & 8 & 0,6 & 0,05 & 2 & 2 & 3 & 0,5 & 0,05 & 2 & 2 & 4 & 0,8 & 0,06 & 1 & 1 & 1 & 0,4 & 0 \\
\hline Malvaceae & 1 & 4 & 50 & 3,8 & 0,75 & 1 & 4 & 43 & 7,6 & 1,64 & 1 & 1 & 5 & 0,9 & 0,08 & 1 & 1 & 2 & 0,8 & 0,13 \\
\hline Nyctaginaceae & 1 & 3 & 37 & 2,8 & 0,32 & 1 & 3 & 7 & 1,2 & 0,17 & 1 & 3 & 17 & 3,2 & 0,36 & 1 & 2 & 13 & 5,0 & 0,59 \\
\hline Limeaceae & 1 & 2 & 14 & 1,1 & 0,15 & 0 & 0 & 0 & 0,0 & 0 & 1 & 2 & 10 & 1,9 & 0,29 & 1 & 1 & 5 & 1,9 & 0,25 \\
\hline Phyllanthaceae & 1 & 2 & 38 & 2,9 & 0,48 & 1 & 1 & 18 & 3,2 & 0,78 & 1 & 2 & 12 & 2,3 & 0,31 & 1 & 2 & 7 & 2,7 & 0,22 \\
\hline Zygophyllaceae & 1 & 1 & 65 & 5,0 & 3,92 & 1 & 1 & 12 & 2,1 & 0,84 & 1 & 1 & 35 & 6,6 & 4,22 & 1 & 1 & 20 & 7,6 & 10,3 \\
\hline Portulacaceae & 1 & 1 & 38 & 2,9 & 0,43 & 1 & 1 & 17 & 3,0 & 0,42 & 1 & 1 & 7 & 1,3 & 0,12 & 1 & 1 & 15 & 5,7 & 1,06 \\
\hline Aizoaceae & 1 & 1 & 27 & 2,1 & 0,32 & 1 & 1 & 14 & 2,5 & 0,57 & 1 & 1 & 9 & 1,7 & 0,19 & 1 & 1 & 3 & 1,1 & 0,13 \\
\hline Acanthaceae & 1 & 1 & 20 & 1,5 & 2,97 & 1 & 1 & 20 & 3,5 & 7,22 & 0 & 0 & 0 & 0,0 & 0 & 1 & 1 & 1 & 0,4 & 0,03 \\
\hline Gisekiaceae & 1 & 1 & 20 & 1,5 & 0,26 & 1 & 1 & 2 & 0,4 & 0,03 & 1 & 1 & 12 & 2,3 & 0,22 & 1 & 1 & 7 & 2,7 & 0,84 \\
\hline Molluginaceae & 1 & 1 & 20 & 1,5 & 0,18 & 1 & 1 & 3 & 0,5 & 0,11 & 1 & 1 & 15 & 2,8 & 0,32 & 1 & 1 & 5 & 1,9 & 0,19 \\
\hline Convolvulaceae & 1 & 1 & 6 & 0,5 & 0,04 & 1 & 1 & 1 & 0,2 & 0,02 & 0 & 0 & 0 & 0,0 & 0 & 1 & 1 & 5 & 1,9 & 0,16 \\
\hline Amaryllidaceae & 1 & 1 & 6 & 0,5 & 0,05 & 0 & 0 & 0 & 0,0 & 0 & 1 & 1 & 3 & 0,6 & 0,07 & 1 & 1 & 3 & 1,1 & 0,03 \\
\hline Araceae & 1 & 1 & 3 & 0,2 & 0,01 & 1 & 1 & 2 & 0,4 & 0,03 & 0 & 0 & 0 & 0,0 & 0 & 0 & 0 & 0 & 0,0 & 0 \\
\hline Commelinaceae & 1 & 1 & 2 & 0,2 & 0,04 & 1 & 1 & 2 & 0,4 & 0,11 & 0 & 0 & 0 & 0,0 & 0 & 0 & 0 & 0 & 0,0 & 0 \\
\hline Pedaliaceae & 1 & 1 & 1 & 0,1 & 0,01 & 1 & 1 & 1 & 0,2 & 0,03 & 0 & 0 & 0 & 0,0 & 0 & 0 & 0 & 0 & 0,0 & 0 \\
\hline Capparaceae & 1 & 1 & 1 & 0,1 & 0,01 & 1 & 1 & 1 & 0,2 & 0,02 & 0 & 0 & 0 & 0,0 & 0 & 0 & 0 & 0 & 0,0 & 0 \\
\hline Total & 41 & 55 & 1312 & 100 & 62,7 & 36 & 47 & 569 & 100 & 82,6 & 33 & 42 & 532 & 100 & 55,33 & 28 & 32 & 262 & 100 & 46,4 \\
\hline
\end{tabular}

Total Genre $(\mathrm{G})$; Espèce $(\mathrm{E})$; Fréquence spécifique (Fs) ; Contribution spécifique $(\mathrm{Cs})$; Recouvrement spécifique (R 
Tableau 2 : Analyse comparative déférentes mares.

\begin{tabular}{|c|c|c|c|c|c|c|c|}
\hline \multirow{2}{*}{\multicolumn{2}{|c|}{ Paramètres statistiques }} & \multirow{2}{*}{ Moyenne } & \multirow{2}{*}{$\begin{array}{l}\text { Ecart } \\
\text { type }\end{array}$} & \multirow{2}{*}{$\begin{array}{l}\text { Coefficient de } \\
\text { Variation (\%) }\end{array}$} & \multicolumn{3}{|c|}{ p-value } \\
\hline & & & & & ML & MT & MS \\
\hline \multirow{3}{*}{ Recouvrement } & ML & 3,8 & 5,0 & 4,1 & - & & \\
\hline & MT & 2,5 & 3,9 & 3,9 & $1,9 \mathrm{E}-11$ & - & \\
\hline & MS & 2,1 & 3,1 & 3,0 & $4,6 \mathrm{E}-7$ & $4,5 \mathrm{E}-10$ & - \\
\hline \multirow{3}{*}{ Contribution } & ML & 4,5 & 5,0 & 2,9 & - & & \\
\hline & MT & 4,5 & 5,5 & 3,4 & $9,8 \mathrm{E}-12$ & - & \\
\hline & MS & 4,5 & 4,8 & 3,1 & 9,2 E-11 & 9,8 E-16 & - \\
\hline
\end{tabular}

Tableau 3 : Composition floristique des herbacées dans chaque mare (Contribution spécifique (Cs) et recouvrement spécifique (Rs)).

\begin{tabular}{lcccccccc}
\hline \multicolumn{1}{c}{ Taxons } & \multicolumn{2}{c}{$\mathbf{1}$} & \multicolumn{2}{c}{$\mathbf{2}$} & \multicolumn{2}{c}{$\mathbf{3}$} \\
\hline Espèces & Cs \% & Rs \% & Cs \% & Rs \% & Cs \% & Rs \% & Cs \% & Rs \% \\
\hline Eragrostis tremula & 8,6 & 13,2 & 9,5 & 21,42 & 7,7 & 8,39 & 8,5 & 6,41 \\
\hline Cyperus esculentus $L$. & 7,5 & 4,7 & 8,1 & 6,09 & 6,0 & 2,9 & 9,3 & 5,78 \\
\hline Indigofera hirsuta L. & 6,5 & 4,01 & 4,2 & 4,22 & 7,3 & 3,59 & 9,7 & 4,66 \\
\hline Zornia glochidiata & 6,3 & 2,82 & 4,7 & 1,32 & 9,0 & 7,15 & 4,8 & 1,09 \\
\hline Tribulus terrestris auct. & 5,0 & 3,92 & 2,1 & 0,85 & 6,6 & 4,22 & 8,1 & 10,25 \\
\hline Enteropogon prieurii & 4,5 & 2,54 & 2,3 & 0,66 & 6,8 & 4,68 & 5,2 & 3,41 \\
\hline Poacées sp & 4,5 & 9,5 & 3,5 & 11,74 & 4,7 & 10,17 & 6,0 & 4,31 \\
\hline Dactyloctenium aegyptium & 3,6 & 1,59 & 1,2 & 0,18 & 5,6 & 2,88 & 4,8 & 2,63 \\
\hline Senna obtusifolia $($ L. $)$ & 3,4 & 0,69 & 6,9 & 1,58 & 1,1 & 0,1 & 0,0 & 0 \\
\hline Alysicarpus ovalifolius & 3,3 & 0,48 & 3,0 & 0,31 & 3,8 & 0,71 & 2,4 & 0,44 \\
\hline Eragrostis ciliaris $($ L. $)$ & 3,0 & 0,89 & 4,4 & 1,65 & 2,6 & 0,49 & 1,6 & 0,38 \\
\hline Portulaca oleracea $L$. & 2,9 & 0,43 & 3,0 & 0,42 & 1,3 & 0,12 & 6,0 & 1,06 \\
\hline Aristida mutabilis & 2,5 & 1,61 & 0,4 & 0,11 & 4,1 & 3,18 & 3,6 & 1,88 \\
\hline Corchorus tridens $L$. & 2,4 & 0,39 & 4,4 & 0,85 & 0,9 & 0,08 & 0,4 & 0,03 \\
\hline Chloris barbata Sw. & 2,3 & 0,63 & 3,9 & 1,29 & 1,5 & 0,27 & 0,0 & 0 \\
\hline Phyllanthus pentandrus & 2,1 & 0,42 & 0,0 & 0 & 1,5 & 0,24 & 0,4 & 0,03 \\
\hline Trianthema pentandra L. & 2,1 & 0,32 & 2,5 & 0,57 & 1,7 & 0,19 & 1,2 & 0,13 \\
\hline Oldenlandia corymbosa & 2,1 & 0,62 & 3,7 & 1,42 & 0,9 & 0,08 & 0,8 & 0,03 \\
\hline Boerhavia erecta L. & 1,6 & 0,16 & 0,7 & 0,06 & 1,7 & 0,2 & 3,2 & 0,31 \\
\hline Spermacoce ruelliae DC. & 1,6 & 1,56 & 3,5 & 3,77 & 0,2 & 0,02 & 0,0 & 0 \\
\hline Peristrophe bicalyculata $(R$ & 1,5 & 2,97 & 3,5 & 7,21 & 0,0 & 0 & 0,0 & 0 \\
\hline & & & & & & & & \\
\hline
\end{tabular}




\begin{tabular}{|c|c|c|c|c|c|c|c|c|}
\hline Gisekia pharnaceoides L. & 1,5 & 0,26 & 0,4 & 0,03 & 2,3 & 0,22 & 2,8 & 0,84 \\
\hline Mollugo nudicaulis Lam & 1,5 & 0,18 & 0,5 & 0,11 & 2,8 & 0,32 & 2,0 & 0,19 \\
\hline Tripogon minimus & 1,5 & 0,29 & 0,2 & 0,02 & 2,4 & 0,64 & 2,4 & 0,25 \\
\hline Brachiaria orthostachys & 1,4 & 1,66 & 3,0 & 4,03 & 0,2 & 0,02 & 0,0 & 0 \\
\hline Achyranthes aspera & 1,3 & 1,04 & 3,0 & 2,49 & 0,0 & 0 & 0,0 & 0 \\
\hline Echinocholoa colona & 1,3 & 1,7 & 1,9 & 3,28 & 1,1 & 0,95 & 0,0 & 0 \\
\hline Cenchrus bifloris & 1,1 & 0,12 & 0,2 & 0,02 & 1,9 & 0,25 & 1,2 & 0,09 \\
\hline Schoenefeldia gracilis & 1,0 & 0,37 & 0,5 & 0,05 & 2,1 & 0,97 & 0,0 & 0 \\
\hline Brachiaria ramosa & 0,9 & 0,35 & 1,2 & 0,32 & 0,2 & 0,02 & 2,0 & 1,03 \\
\hline Boerhavia diffusa & 0,8 & 0,1 & 0,4 & 0,03 & 0,8 & 0,08 & 2,0 & 0,28 \\
\hline Digitaria horizontalis & 0,8 & 0,08 & 0,5 & 0,05 & 1,5 & 0,15 & 0,8 & 0,06 \\
\hline Oldenlandia sp & 0,8 & 0,07 & 0,7 & 0,06 & 1,1 & 0,1 & 0,8 & 0,06 \\
\hline Achyranthes sp & 0,8 & 1,63 & 1,8 & 3,91 & 0,0 & 0 & 0,0 & 0 \\
\hline Limeum viscosum & 0,8 & 0,09 & 0,0 & 0 & 1,1 & 0,14 & 2,0 & 0,25 \\
\hline Phyllanthus niruri auct. & 0,8 & 0,06 & 3,2 & 0,78 & 0,8 & 0,07 & 2,4 & 0,19 \\
\hline Corchorus sp & 0,7 & 0,16 & 1,6 & 0,38 & 0,0 & 0 & 0,0 & 0 \\
\hline Brachiaria Lata & 0,6 & 0,22 & 1,4 & 0,54 & 0,0 & 0 & 0,0 & 0 \\
\hline Fimbristylis hispidula & 0,5 & 0,28 & 0,0 & 0 & 1,9 & 1,2 & 0,0 & 0 \\
\hline Corchorus olitoris & 0,5 & 0,16 & 1,1 & 0,35 & 0,0 & 0 & 1,2 & 0,09 \\
\hline Pancratium trianthum auct. & 0,5 & 0,05 & 0,0 & 0 & 0,6 & 0,07 & 1,2 & 0,13 \\
\hline Ipomoea coccinea & 0,5 & 0,04 & 0,2 & 0,02 & 0,0 & 0 & 2,0 & 0,16 \\
\hline Oldenlandia linearis DC. & 0,5 & 0,04 & 0,0 & 0 & 1,1 & 0,1 & 0,0 & 0 \\
\hline Spermacoce sp & 0,5 & 0,04 & 0,7 & 0,06 & 0,6 & 0,05 & 0,0 & 0 \\
\hline Cucumis melo var. agrestis & 0,4 & 0,03 & 0,4 & 0,03 & 0,4 & 0,03 & 0,4 & 0,03 \\
\hline Boerhavia sp & 0,4 & 0,06 & 0,2 & 0,08 & 0,8 & 0,07 & 0,0 & 0 \\
\hline Limeum indicum & 0,3 & 0,06 & 0,0 & 0 & 0,8 & 0,15 & 0,0 & 0 \\
\hline Stylochaeton hypogaeus & 0,2 & 0,01 & 0,4 & 0,03 & 0,0 & 0 & 0,0 & 0 \\
\hline Momordica charantia L. & 0,2 & 0,02 & 0,2 & 0,02 & 0,4 & 0,03 & 0,0 & 0 \\
\hline Corchorus aestuans & 0,2 & 0,03 & 0,5 & 0,06 & 0,0 & 0 & 0,0 & 0 \\
\hline Commelina forsskaolii Vahl & 0,2 & 0,04 & 0,4 & 0,11 & 0,0 & 0 & 0,0 & 0 \\
\hline Amaranthus hybridus L. & 0,1 & 0,01 & 0,0 & 0 & 0,0 & 0 & 0,4 & 0,03 \\
\hline Cleome viscosa $L$. & 0,1 & 0,01 & 0,2 & 0,02 & 0,0 & 0 & 0,0 & 0 \\
\hline Kilinga brevifolia & 0,1 & 0,01 & 0,0 & 0 & 0,2 & 0,02 & 0,0 & 0 \\
\hline Sesamum sp & 0,1 & 0,01 & 0,2 & 0,03 & 0,0 & 0 & 0,0 & 0 \\
\hline 55 & 100 & 62,73 & 100 & 82,63 & 100 & 55,31 & 100 & 46,51 \\
\hline
\end{tabular}

1 : Zone ; 2 : Mare aux lions ; 3 : Mare à Trou ; 4 : Mare de Belel Sow 
Tableau 4 : Nombre de genres $(\mathrm{G})$ et espèces $(\mathrm{E})$, fréquences $(\mathrm{Fs})$, contributions $(\mathrm{Cs})$ et recouvrements spécifiques (Rs) des familles rencontrées sur les différentes unités topographiques.

\begin{tabular}{|c|c|c|c|c|c|c|c|c|c|c|c|c|c|c|c|}
\hline \multirow{3}{*}{$\begin{array}{l}\text { Années } \\
\text { Familles }\end{array}$} & \multicolumn{5}{|c|}{ Mare } & \multicolumn{5}{|c|}{ Versant } & \multicolumn{5}{|c|}{ Plateau } \\
\hline & \multicolumn{2}{|c|}{$\begin{array}{c}\text { Nombre } \\
\text { de }\end{array}$} & \multirow[t]{2}{*}{ Fs } & \multirow{2}{*}{$\begin{array}{l}\text { Cs } \\
\%\end{array}$} & \multirow{2}{*}{ Rs \% } & \multicolumn{2}{|c|}{ Nombre de } & \multirow[t]{2}{*}{ Fs } & \multirow{2}{*}{$\begin{array}{l}\text { Cs } \\
\%\end{array}$} & \multirow[t]{2}{*}{ Rs \% } & \multicolumn{2}{|c|}{ Nombre de } & \multirow[t]{2}{*}{ Fs } & \multirow{2}{*}{$\begin{array}{l}\text { Cs } \\
\%\end{array}$} & \multirow[t]{2}{*}{ Rs \% } \\
\hline & $G$ & $E$ & & & & $\mathbf{G}$ & $\mathbf{E}$ & & & & $\mathbf{G}$ & $\mathbf{E}$ & & & \\
\hline Poaceae & 11 & 14 & 139 & 35,4 & 41,63 & 12 & 15 & 197 & 35,3 & 37,2 & 10 & 11 & 146 & 43,8 & 20,27 \\
\hline Fabaceae & 4 & 4 & 85 & 21,6 & 5,59 & 4 & 4 & 101 & 18,1 & 6,89 & 4 & 4 & 71 & 21,3 & 13,39 \\
\hline Rubiaceae & 2 & 3 & 15 & 3,8 & 2,44 & 2 & 5 & 36 & 6,5 & 3,39 & 2 & 5 & 21 & 6,3 & 0,61 \\
\hline Amaranthaceae & 2 & 2 & 5 & 1,3 & 1,02 & 2 & 3 & 21 & 3,8 & 5,67 & 1 & 2 & 2 & 0,6 & 0,16 \\
\hline Cyperaceae & 1 & 1 & 36 & 9,2 & 6,36 & 3 & 3 & 52 & 9,3 & 5,81 & 2 & 2 & 19 & 5,7 & 2,38 \\
\hline Acanthaceae & 1 & 1 & 7 & 1,8 & 0,42 & 1 & 1 & 13 & 2,3 & 7,1 & 0 & 0 & 0 & 0,0 & 0 \\
\hline Aizoaceae & 1 & 1 & 11 & 2,8 & 0,3 & 1 & 1 & 13 & 2,3 & 0,02 & 0 & 0 & 0 & 0,0 & 0 \\
\hline Gisekiaceae & 1 & 1 & 2 & 0,5 & 0,04 & 1 & 1 & 9 & 1,6 & 0,32 & 1 & 1 & 9 & 2,7 & 0,48 \\
\hline Limeaceae & 1 & 1 & 2 & 0,5 & 0,13 & 1 & 2 & 4 & 0,7 & 0,1 & 0 & 0 & 0 & 0,0 & 0 \\
\hline Malvaceae & 1 & 4 & 22 & 5,6 & 1 & 1 & 4 & 26 & 4,7 & 0,95 & 1 & 4 & 2 & 0,6 & 0,11 \\
\hline Nyctaginaceae & 1 & 3 & 14 & 3,6 & 0,38 & 1 & 2 & 13 & 2,3 & 0,97 & 1 & 3 & 13 & 3,9 & 0,98 \\
\hline Pedaliaceae & 1 & 1 & 1 & 0,3 & 0,04 & 0 & 0 & 0 & 0,0 & 0 & 0 & 0 & 0 & 0,0 & 0 \\
\hline Phyllanthaceae & 1 & 2 & 20 & 5,1 & 0,74 & 1 & 2 & 14 & 2,5 & 0,54 & 0 & 0 & 0 & 0,0 & 0 \\
\hline Portulacaceae & 1 & 1 & 13 & 3,3 & 0,43 & 1 & 1 & 19 & 3,4 & 0,57 & 1 & 1 & 7 & 2,1 & 0,23 \\
\hline Zygophyllaceae & 1 & 1 & 16 & 4,1 & 2,7 & 1 & 1 & 22 & 3,9 & 3,11 & 1 & 1 & 29 & 8,7 & 7,08 \\
\hline Araceae & 1 & 1 & 1 & 0,3 & 0,02 & 1 & 1 & 1 & 0,2 & 0,02 & 0 & 0 & 0 & 0,0 & 0 \\
\hline Convolvulaceae & 1 & 1 & 1 & 0,3 & 0,02 & 1 & 1 & 3 & 0,5 & 0,05 & 1 & 1 & 2 & 0,6 & 0,05 \\
\hline Molluginaceae & 1 & 1 & 3 & 0,8 & 0,06 & 1 & 1 & 6 & 1,1 & 0,17 & 1 & 1 & 1 & 0,3 & 0,35 \\
\hline Cucurbitaceae & 0 & 0 & 0 & 0,0 & 0 & 2 & 2 & 5 & 0,9 & 0,08 & 2 & 2 & 2 & 0,6 & 0,33 \\
\hline Amaryllidaceae & 0 & 0 & 0 & 0,0 & 0 & 1 & 1 & 2 & 0,4 & 0,05 & 1 & 1 & 7 & 2,1 & 0,2 \\
\hline Capparaceae & 0 & 0 & 0 & 0,0 & 0 & 0 & 0 & 0 & 0,0 & 0 & 1 & 1 & 1 & 0,3 & 0,03 \\
\hline Commelinaceae & 0 & 0 & 0 & 0,0 & 0 & 1 & 1 & 1 & 0,2 & 0,1 & 1 & 1 & 1 & 0,3 & 0,03 \\
\hline Total & 33 & 43 & 393 & 100 & 63,32 & 39 & 52 & 558 & 100 & 73,1 & 31 & 41 & 333 & 100 & 46,68 \\
\hline
\end{tabular}

Tableau 5 : Analyse comparative déférentes unités topographique.

\begin{tabular}{|c|c|c|c|c|c|c|c|}
\hline \multirow{2}{*}{\multicolumn{2}{|c|}{ Paramètres statistiques }} & \multirow{2}{*}{ Moyenne } & \multirow{2}{*}{$\begin{array}{l}\text { Ecart } \\
\text { type }\end{array}$} & \multirow{2}{*}{$\begin{array}{l}\text { Coefficient de } \\
\text { Variation }(\%)\end{array}$} & \multicolumn{3}{|c|}{ p-value } \\
\hline & & & & & Mare & Versant & Plateau \\
\hline \multirow{3}{*}{ Recouvrement } & Mare & 2,9 & 8,8 & 4,4 & - & & \\
\hline & Versant & 3,3 & 7,9 & 4,0 & $7,9 \mathrm{E}-15$ & - & \\
\hline & Plateau & 2,1 & 5,1 & 2,9 & $1,9 \mathrm{E}-7$ & $3,6 \mathrm{E}-7$ & - \\
\hline \multirow{3}{*}{ Contribution } & Mare & 4,5 & 8,4 & 3,0 & - & & \\
\hline & Versant & 4,5 & 8,0 & 3,2 & 2,2 E-16 & - & \\
\hline & Plateau & 4,5 & 10,0 & 3,4 & 7,2 E-14 & $2,1 \mathrm{E}-14$ & - \\
\hline
\end{tabular}


Tableau 6 : Indices de similitude entre les sites.

\begin{tabular}{|c|c|c|c|c|c|c|c|c|c|}
\hline & \multicolumn{2}{|c|}{ Mares } & \multicolumn{2}{|c|}{ Mare Belel Sow } & Mare à Trous & \multicolumn{2}{|c|}{ Mare aux lions } & & \\
\hline & \multicolumn{2}{|c|}{ Mare Belel Sow } & \multicolumn{2}{|l|}{ - } & 0,64 & \multicolumn{2}{|c|}{0,56} & & \\
\hline & \multicolumn{2}{|c|}{ Mare à Trous } & \multicolumn{2}{|l|}{0,64} & - & \multicolumn{2}{|c|}{0,69} & & \\
\hline & \multicolumn{2}{|c|}{ Mare aux lions } & 0,56 & \multicolumn{2}{|r|}{0,69} & \multicolumn{2}{|l|}{ - } & & \\
\hline Zones & $\begin{array}{l}\text { Fond } \\
\text { Mare }\end{array}$ & $\begin{array}{c}\text { Zone } \\
\text { transition }\end{array}$ & $\begin{array}{l}\text { Inter } \\
\text { dune }\end{array}$ & $\begin{array}{l}\text { Fond } \\
\text { Mare }\end{array}$ & $\begin{array}{c}\text { Zone } \\
\text { transition }\end{array}$ & $\begin{array}{l}\text { Inter } \\
\text { dune }\end{array}$ & $\begin{array}{l}\text { Fond } \\
\text { Mare }\end{array}$ & $\begin{array}{c}\text { Zone } \\
\text { transition }\end{array}$ & $\begin{array}{l}\text { Inter } \\
\text { dune }\end{array}$ \\
\hline Fond Mare & - & 0,48 & 0,54 & - & 0,7 & 0,54 & - & 0,59 & 0,42 \\
\hline Zone transition & 0,48 & - & 0,7 & 0,7 & - & 0,63 & 0,59 & - & 0,55 \\
\hline Interdune & 0,54 & 0,7 & - & 0,54 & 0,63 & - & 0,42 & 0,55 & - \\
\hline
\end{tabular}

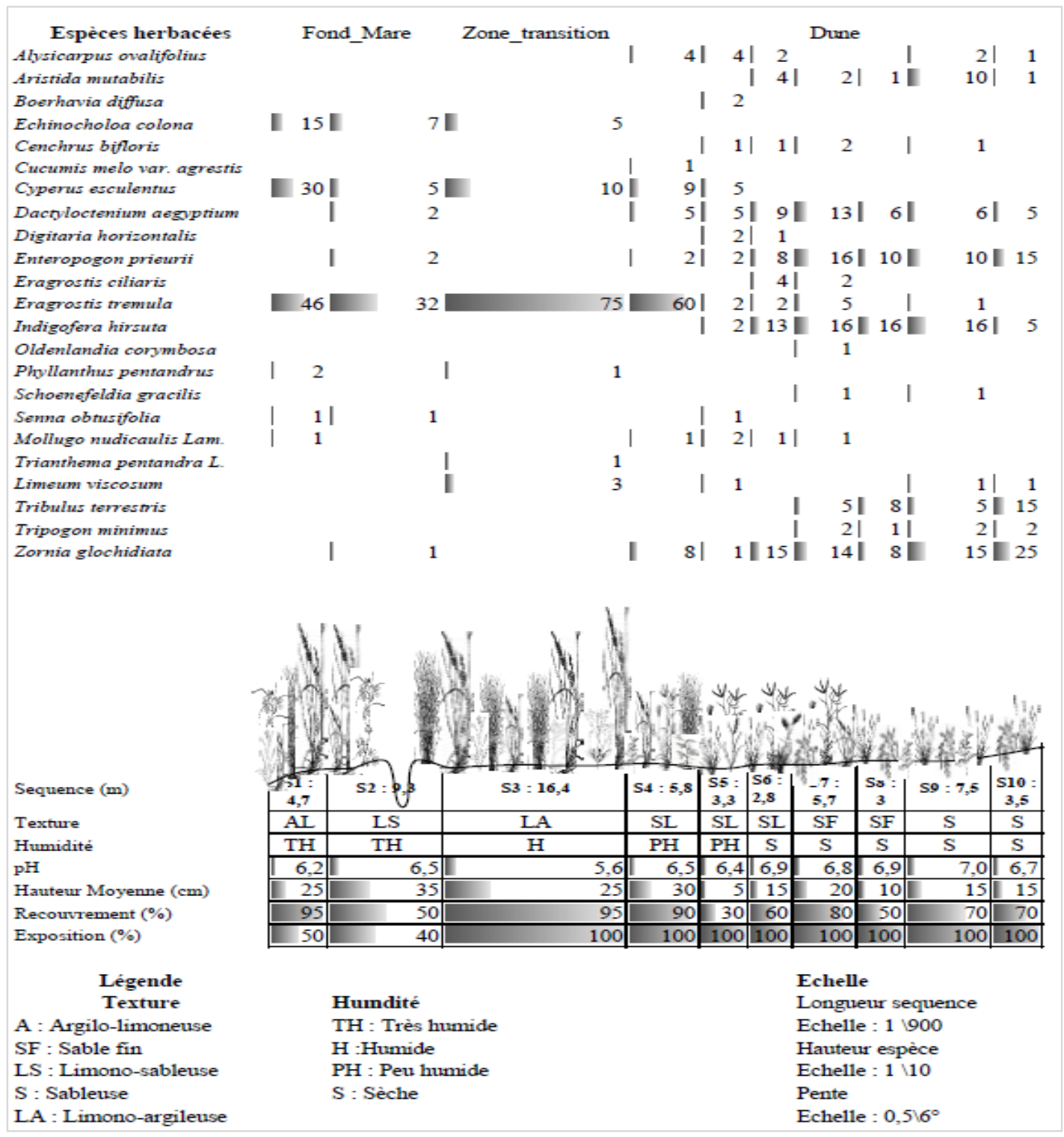

Figure 5 : Transect phytoécologique de la mare à Trou. 


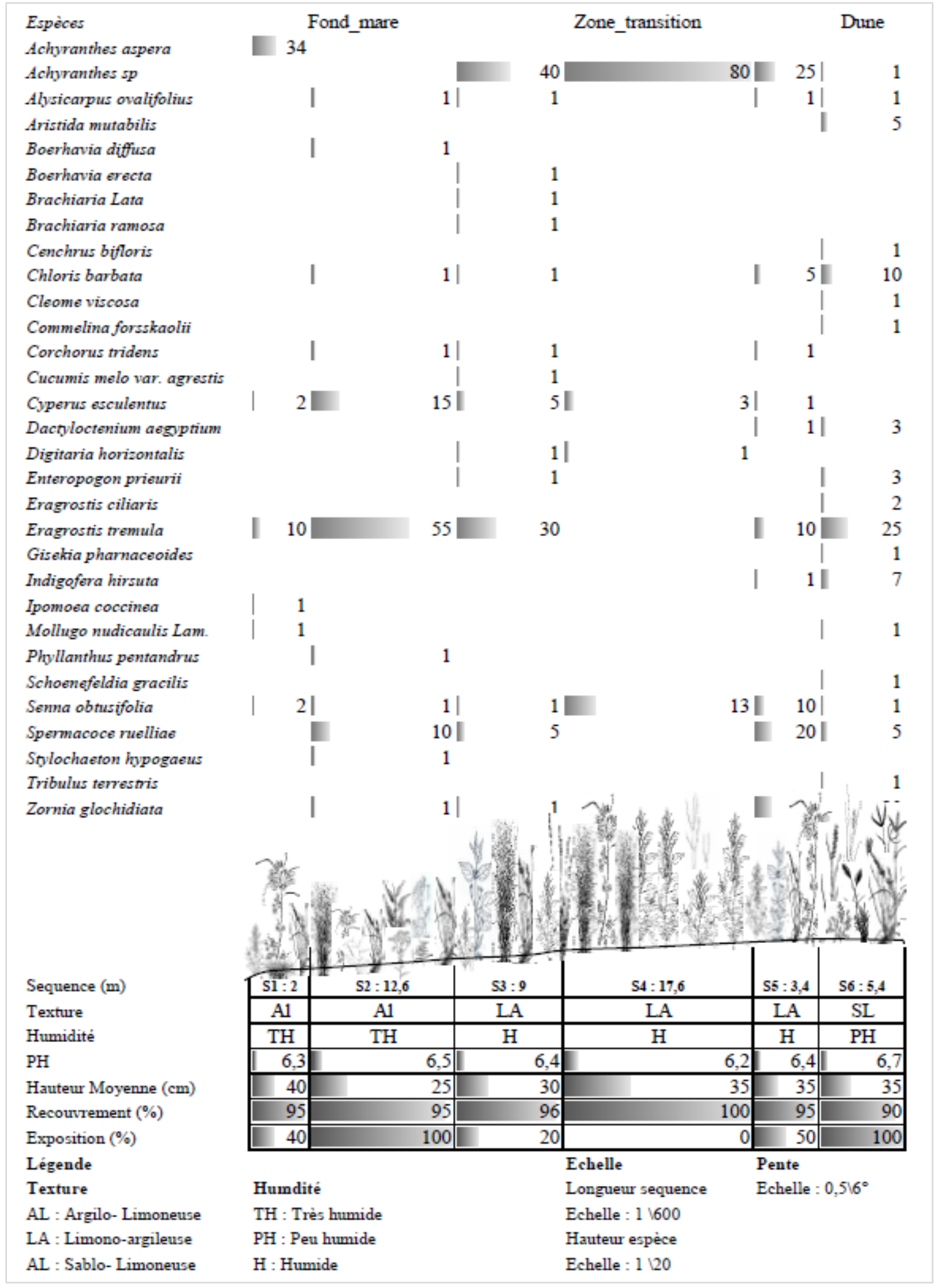

Figure 6 : Transect phytoécologique de la mare aux lions. 


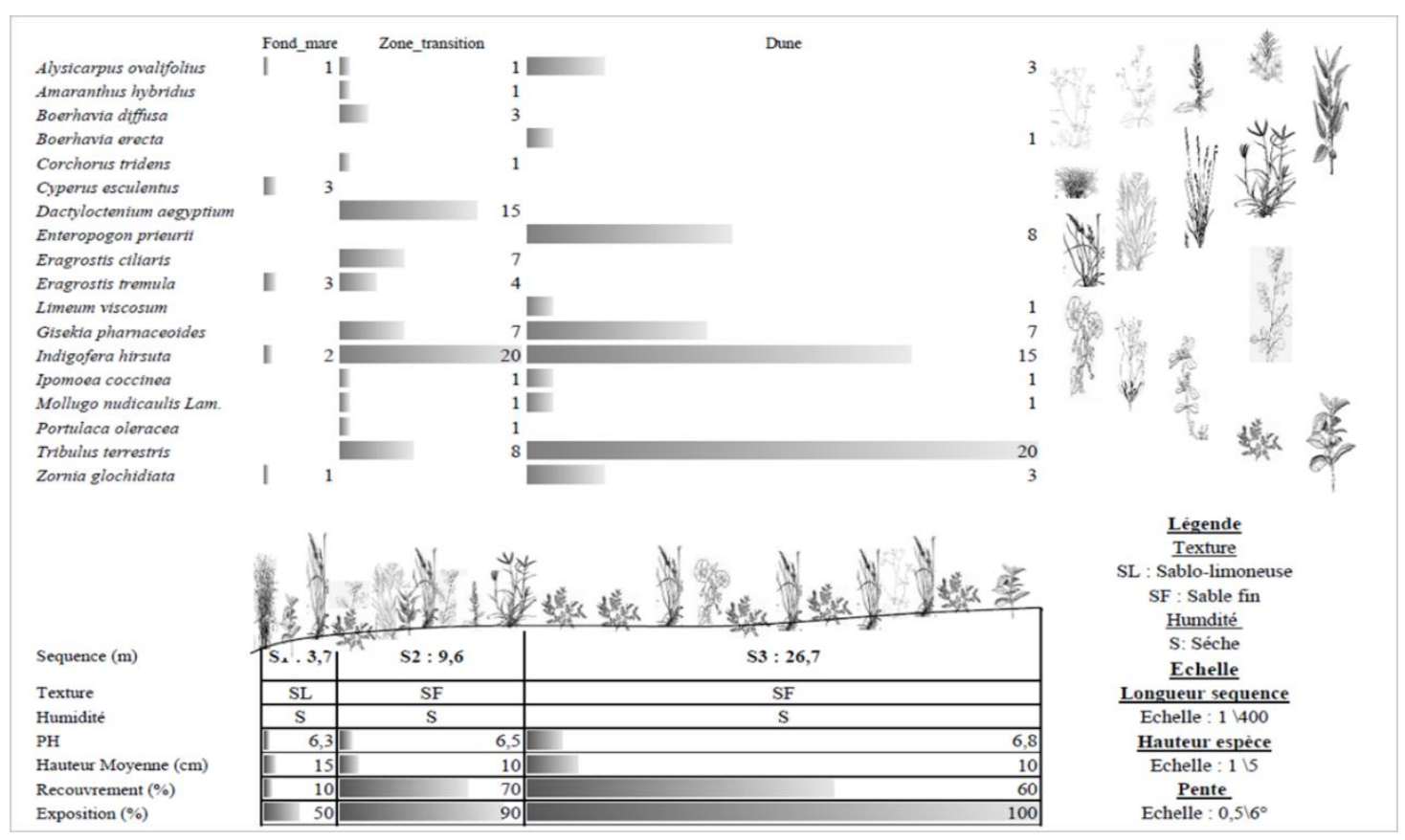

Figure 7. Transect phytoécologique de la mare de Belel Sow.

\section{DISCUSSION}

Le cortège floristique de la strate herbacée étudiée autour des mares temporaires comprend 55 espèces appartenant à 41 genres inféodées dans 22 familles. La famille la plus représentative est les Poacées, suivi la famille des Fabacées et la famille des Rubiacées. La forte présence des Poacées dans la zone d'étude peut s'expliquer par le fait que ces taxons possèdent une très grande possibilité de repousse. Ces résultats concordent avec ceux de (Ndiaye et al., 2013; Yoka etal., 2013 ; Diallo et al., 2015).

Cette flore n'est pas homogène dans toutes les mares étudiées puisque des disparités se présentent d'une mare à l'autre. En effet le cortège floristique de la mare aux lions se distingue de celui de la mare de Sow et la mare à Trou par une forte richesse et par la présence d'un petit groupe d'espèces propre à cette mare. Ce groupe est constitué d'ombrophytes (Peristrophe bicalyculata, Achyranthes aspera, Brachiaria Lata, Corchorus sp, Commelin aforsskaolii Vahl) qui s'y développent en formation dense et élevée. Une telle communauté peut être traduite par une forte présence des arbres et la nature du substrat (Diallo et al., 2006; Remigi et al., 2008 ; Diallo et al., 2015). Par contre, le cortège floristique des deux mares (mare de Belel Sow et mare à Trou) apparaît dominer par des espèces de la famille des Poacées et des Zygophyllacées dont la présence indique une dégradation de la structure du sol (Ousseina et al., 2013). La mare à Trou et la mare de Belel Sow présentent des conditions environnementales défavorables aux développements de certaines espèces. Au fait une forte fréquentation en saison pluvieuse est observée dans ces mares entraînant une dégradation importante du couvert végétal. Il faut noter que la mare est un écosystème original, dont le fonctionnement est intimement lié au milieu environnant (Jourdas, 2013) ainsi la mare aux lions est clôturée depuis des années via les projets allemands. De plus, les agents de la grande muraille verte ont mis une autre clôture de notre zone expérimentale en 2018. La mare évolue donc naturellement avec l'absence de perturbation (ici le pâturage) et l'action anthropique qui y est très faible. 
D'ailleurs l'indice de similitude montre une différence marquante entre la mare aux lions non pâturée et la mare de Belel Sow pâturée régulièrement $(0,56)$. L'influence du pâturage a été démontrée dans la mare de Belel Sow par la petite taille des plantes, la présence de plages de sol nu (Jamaa et al., 2014). Le pâturage devient un des facteurs de contrôle de la composition floristique et de la stratification végétale des mares étudiées.

En considérant les différentes unités topographiques, la composition floristique est assez diversifiées en espèces herbacées quel que soit la toposéquence. Selon Diallo et al. (2013), la flore d'une station (richesse floristique ou diversité $\alpha$ ) est considérée comme 'assez riche' lorsqu'elle renferme 31 à 40 espèces. La comparaison de la composition floristique des différentes unités topographique montre que les versants renferment une plus grande diversité et la plus forte densité. Cela peut être expliqué par le fait que le versant est la zone de transition entre la zone inondée et la zone exondée, beaucoup d'espèces $\mathrm{y}$ s'adaptent.

En plus la comparaison des différents transects phytoécologies montrent que les zones de transition ont une grande diversité et de fort recouvrement quel que soit la mare. Elles restent plus souvent les zones à faible exposition par rapport au soleil.

Les transects phytoécologies montrent aussi que l'espèce Tribulus terrestris est très abondante dans la partie exondée de la mare de Belel Sow et de la mare à Trou. Ce sont les zones de textures sableuses, sèches, dépourvus d'arbres et surpâturées. En revanche l'espèce Achyranthes $s p$ se trouve dans les zones à humidité intermédiaire avec une forte présence des ligneux. La mare aux lions présente beaucoup de zones favorables au développement de nombreuses espèces. Il faut noter que la présence de l'arbre est bénéfique pour le développement des espèces ce qui est démontré par Akpo et Grouzis (2009) dans les écosystèmes sahéliens. De plus selon Akpo et Grouzis (2004) dans les zones protégées, l'arbre exerce un effet favorable sur la végétation herbacée, par rapport au biotope découvert, car il améliore la diversité spécifique et la production de phytomasse herbacée. Cet effet est nettement plus faible que dans les milieux régulièrement pâturés (mare de Belel Sow).

\section{Conclusion}

La présente étude a permis de faire une évaluation de la répartition spatiale et la structure de la végétation herbacée autour des mares temporaires au Ferlo. La comparaison entre les données floristiques recueillies dans une mare temporaire non pâturée et dans deux mares pâturées a permis de démontrer l'influence de l'action anthropique sur la végétation herbacée autour de cet écosystème au Ferlo, et de mettre en évidence le rôle de la topographique. Les résultats obtenus révèlent l'influence prédominante du pâturage et de la topographique sur la structuration, la stratification verticale de la végétation et de leur extension spatiale. A l'issue de cette étude, il est important de maintenir une mosaïque paysagère comprenant des mares non pâturées et des mares pâturées réservées exclusivement à l'abreuvement du bétail. Cette étude sera succédée par une étude sur l'influence des facteurs éco-hydrologique sur la biodiversité de la végétation herbacée autour des mares et une étude qui évalue les paramètres physicochimiques du sol.

\section{CONFLIT D'INTERETS}

Les auteurs déclarent qu'ils n'ont aucun conflit d'intérêts concernant cet article.

\section{CONTRIBUTIONS DES AUTEURS}

$\mathrm{NF}$ : travail de terrain, rédaction du manuscrit, confection des cartes et des figures ; $\mathrm{AD}$ : travail de terrain, confection des cartes et des figures et correction du manuscrit; MBS : travail de terrain, confection des cartes et des figures et correction du manuscrit; OS : travail de terrain; RT: travail de terrain; ESB : travail de terrain ; JLP : conception de cartes ; AG : Auteur correspondant, coordination des thèmes de recherche, correction et soumission.

\section{REMERCIEMENTS}

Nous remercions le projet

"Observatoire international homme- 
environnement (OHMi)" de Téssékéré pour assurer la logistique sur le terrain.

\section{REFERENCES}

Akpo LE, Grouzis M. 2004. Interactions arbre/herbe en bioclimat semi-aride: Influence de la pâture. Sécheresse, 15(3): 253-261.

Akpo LE, Grouzis M. 2009. Effet des arbres sur la diversité de la végétation herbacée dans les parcours communautaires du Nord-Sénégal (Afrique de l'Ouest). Jour. Agri. Envi. Inter. Dev., 103(4): 271-293. DOI: https://doi.org/10.12895/jaeid.20094.36

Bakhoum A. 2013. Dynamique des ressources fourragères: indicateur de résilience des parcours communautaires de Téssékére au Ferlo, (Nord-Sénégal). Thèse de doctorat. Biologie, Productions et Pathologies Animales. Université cheikh Anta Diop de Dakar. p. 118.

Cissé S, Eymard L, Ndione JA, Gaye AT. 2015. Analyse statistique des relations pluie-végétation au Ferlo (Sénégal). XXVIIIe Colloque de l'Association Internationale de Climatologie, Liège.307-312.

Diallo A, Faye M, Ndiaye O, Guisse A. 2011. Variations de la composition de la végétation herbacée des plantations d'Acacia senegal $($ L.) Willd de la zone de Dahra (Ferlo). Int. J. Biol. Chem. Sci., 5(3): $\quad 1250-1264 . \quad$ DOI: 10.4314/ijbcs.v5i3.72273

Diallo A, Guisse A, Ngom Faye M, Saradoum G. 2009. Variabilité floristique de la végétation herbacée de la Niaye de Pikine au Sénégal. Rev. Écol. (Terre Vie), 64(2009) : 123-133. URL : http://hdl.handle.net/2042/55781

Diallo H, Faye EH, Kone B, Bindelle J, Lejoly J, Maiga M. 2013. Biodiversité et valeur pastorale des herbacées de la Réserve de Fina (Mali). Scripta Botanica Belgica. 50: $111-120$.

Diallo MD, Duponnois R., Guisse A, Sall S, Chotte JL, Thioulouse J. 2006. Biological effects of native and exotic plant residues on plant growth, microbial biomass and availability under controlled conditions. European Journal of Soil Biology, 42(4):
238-246.

DOI:https://doi.org/10.1016/j.ejsobi.2005 .01 .003

Diallo MD, Ngamb T, Tine AK, Guisse M, Ndiaye O, Saleh MM, Diallo A, Seck S, Diop A, Guisse A. 2015. Caractérisation agropédologique des sols de MBOLTIME dans la zone des NIAYES (Sénégal). Agronomie Africaine, 27 (1) : $57-67$.

Diallo M, Saleh M, Bassene C, Wood S, Diop A, Guisse A. 2015. Influence de la litière foliaire de cinq espèces végétales tropicales sur la diversité floristique des herbacées dans la zone du Ferlo (Sénégal). Int. J. Biol. Chem. Sci., 9(2): 803-814. DOI: 10.4314/ijbcs.v9i2.20

Diop AT, Diaw OT, Dieme I, Touré I, Sy O, Diémé G. 2004. Mares de la zone sylvopastorale du Sénégal : Tendances évolutives et rôle dans les stratégies de production des populations pastorales. Revue d'Elevage et de Médecine Vétérinaire des Pays Tropicaux, 57(1-2): 77-85.

DOI:https://doi.org/10.19182/remvt.9910

Fournet J, Monestiez P. 1987. Essai de caractérisation phytoécologique des formations herbacées pâturées de GrandeTerre (Guadeloupe). Agronomie, 7(10): 833-851. DOI: https://hal.archivesouvertes.fr/hal-00884960

Jamaa FB, Mokhtar A, Saad B. 2014. Influence du pâturage sur la structure, la composition et la dynamique de la végétation de mares temporaires méditerranéennes (Tunisie septentrionale). Rev. Écol. (Terre Vie), 69: 196-213. URL: http://hdl.handle.net/2042/56021

Jourdas A. 2013. Comprendre la mare à travers sa biodiversité. (VALMARES, UN PROJET AUTOUR DES MARES) .p. 20.

Ka A. 2016. Manger à WIDOU THIENGOLY (Nord-Sénégal): De l'abondance remémorée à la dépendance au marché. Thèse de doctorat; Spécialité : Anthropologie de l'alimentation. Université Cheikh Anta Diop de Dakar. p. 330. 
Ndiaye I, Camara B, Ngom D. 2017. Diversité spécifique et usages ethnobotaniques des ligneux suivant un gradient pluviométrique Nord-Sud dans le bassin arachidier sénégalais. J.Appl. Biosci., 113: $11123-11137 . \quad$ DOI: https://dx.doi.org/10.4314/jab.v113i1.2

Ndiaye M, Dione ME, Akpo LE. 2010. Caractéristiques des ligneux dans les Terroirs pastoraux de Ranérou (Région de Matam, Nord-Sénégal). Journal des Sciences, 3: 12-27. http://www.cadjds.org Ndiaye O, Diop A, Akpo LE, Diéne M. 2014. Dynamique de la teneur en carbone et en azote des sols dans les systèmes d'exploitation du Ferlo: Cas du CRZ de Dahra. Jour. Appl Biosci., 83 : 7554 7569. DOI: 10.4314 jab.v83i1.5

Ndiaye O. 2015. Déterminants de la dynamique de la végétation d'un milieu pâture en région sahélienne du Sénégal. Thèse de doctorat en Biologie, Productions et Pathologies Animales. Université Cheikh Anta Diop de Dakar. p. 138.

Ndiaye O, Diallo A, Sagna MB, Guisse A. 2013. Diversité floristique des peuplements ligneux du Ferlo, Sénégal. VertigO, 13(3): 1-12. DOI: https://doi.org/10.4000/vertigo.14352

Ngom D, Bakhoum A, Diatta S, Akpo LE. 2012. Qualité pastorale des ressources herbagères de la réserve de biosphère du Ferlo (Nord-Sénégal). Int. J. Biol. Chem. Sci., 6(1): 186-201. DOI: 10.4314/ijbcs.v6i1.17

Niang K, Ndiaye O, Diallo A, Guisse, A. 2014. Flore et structure de la végétation ligneuse le long de la Grande Muraille Verte au Ferlo, nord Sénégal. Jour. Appl. Bioscie, 79(1): 6938-6946. DOI: https://doi.org/10.4314/jab.v79i1.15

Ousseina S, Fortina R, Marichatou $\mathrm{H}$, Tenikoye A. 2013. Dynamique du peuplement herbacé de la station sahélienne expérimentale de Toukounous (Filingué - Niger). Int. J. Biol. Chem. Sci., 7(2): 657-671. DOI: https://doi.org/10.4314/ijbcs.v7i2.22

Pierre C, Bergametti G, Marticorena B, Mougin E, Lebel T, Ali A. 2011.Pluriannual comparisons of satellite-based rainfall products over the Sahelian belt for seasonal vegetation modeling. Jou. Geo. Resea, 116(18): 125 . DOI: https://doi.org/10.1029/2011JD016115

Remigi P, Faye A, Kane A, Deruaz M, Thioulouse J, Cissoko M., Prin Y, Galiana A, Dreyfu B, Duponnois R. 2008. The exotic legume tree species Acacia holosericea alters microbial soil functionalities and the structure of the Arbuscular Mycorrhizal Community. Appl. Envi. Micro., 74(5): 1485-1493. DOI: https://doi.org/10.1128/AEM.02427-07

Sarr O, Diatta S, Gueye M, Ndiaye PM, Guisse A, Akpo LE. 2013. Importance des ligneux fourragers dans un système agropastoral au Sénégal (Afrique de l'ouest). Revue Méd. Vét., 164(2013) : 28. DOI: https://hal.archivesouvertes.fr/hal-01722601

Sy O. 2009. Rôle de la mare dans la gestion des systèmes pastoraux sahéliens du Ferlo (Sénégal). Cybergeo: Eu. Jour. Geo., 440: $1-11$. DOI: $10.4000 /$ cybergeo. 22057

Yoka J, Loumeto JJ, Djego J, Vouidibio J. 2013. Évaluation de la diversité floristique en herbacées des savanes de la cuvette congolaise (République du Congo). Afrique SCIENCE, 9(2): $110-$ 123.

DOI: http://www.afriquescience.info/documen t.php?id=2831. 